\title{
RECENT DEVELOPMENTS IN ULTRASONOGRAPHY
}

\author{
LEONARDO MASOTTI *
}

ABSTRACT. - Developments in high resolution probes, data processing techniques and contrast means are providing a new generation of ultrasound technologies for diagnostic purposes. In this paper new results are presented derived from research performed at the Ultrasound Laboratory of the University of Florence. A new hardware and software platform called Fast Ecographic Multi Image Novel Apparatus (FEMMINA) has been developed based on innovative features. The radiofrequency signals, directly provided by the US transducers, contains fundamental information on the linear and non linear interaction of US with biological tissues.

New suitable algorithms have been therefore developed to process on line such radiofrequency signals in order to identify and present information of clinical value. The main concept is the quantification of suitable parameters characterizing the morphology of the local frequency spectrum derived from a little volume of the biological tissue under investigation. Such parameters could be correlated to the microstructure tissue properties and are presented on the traditional tomographic map which are normally present in ultrasound instrumentation. The clinical validation of these techniques are illustrated pointing out the new perspectives for a more efficient and detailed analysis for selected pathologies.

* Dipartimento di Elettronica e Telecomunicazioni - Laboratorio Ultrasuoni e Controlli non Distruttivi - Università degli Studi di Firenze. 


\section{PREMESSA}

Si riportano nel presente scritto risultati dell'attività del nostro Laboratorio di Firenze, impegnato da molti anni, anche in collaborazione con ESAOTE, nello sviluppo di ricerche su dispositivi, metodi e sistemi per la diagnosi medica mediante ultrasuoni. Il gruppo del nostro Laboratorio è costituito dalle seguenti persone: Leonardo Masotti, Elena Biagi, Lorenzo Capineri, Simona Granchi, Marco Scabia, Alberto Acquafresca, Luca Breschi, Rodolfo Facchini, Stefano Mazzanti, Andrea Ricci, Francesco Rindi, Jonatan Torricelli.

\section{INTRODUZIONE}

Nella moderna medicina si tende sempre più a fare uso di immagini di significato diagnostico. In senso generale le immagini consistono in una rappresentazione sintetica di informazioni portate da una sorgente di energia che può essere generata dall'oggetto sotto osservazione o può essere messa a disposizione da una sorgente opportuna.

Per il corpo umano si possono creare immagini di organi o della loro attività utilizzando energia da questo emessa naturalmente: segnali elettrici (tracciati e mappe elettrocardiografici ed elettroencefalografici), segnali magnetici (magnetocardiogrammi), raggi infrarossi (termovisione), segnali fonici (fonogrammi), o facendo uso di energia iniettata (scintigrafia); altri sistemi: endoscopia, radiografia, ecografia, indagini mediante risonanza magnetica nucleare, richiedono l'intervento di sorgenti di energia esterne.

Nelle indagini con energia emessa da sorgenti esterne quali i raggi $\mathrm{X}$, gli ultrasuoni, prodotti radioattivi iniettati, le informazioni contenute nelle immagini sono determinate dalle interazioni che avvengono tra l'energia e l'organo in esame. L'interazione tra l'organo e l'energia può essere visualizzata in due differenti modi; può essere creata un'immagine mediante l'energia che riesce a passare attraverso l'organo o sue porzioni, oppure può essere trasformata in immagini l'energia che viene riflessa o diffusa da porzioni della struttura in esame.

Le due tecniche sono chiamate rispettivamente visualizzazione per trasmissione e visualizzazione per riflessione.

Nella tecnica di visualizzazione per trasmissione l'energia si propaga attraverso l'oggetto, cioè i tessuti biologici: l'energia che non viene 
assorbita, diffusa o riflessa dai tessuti è visualizzata. La radiografia convenzionale impiega questa tecnica.

La tecnica di visualizzazione per riflessione utilizza l'energia che è stata retrodiffusa o riflessa dai tessuti. L'ecografia ad ultrasuoni attualmente utilizza nella maggior parte dei casi la tecnica di formazione delle immagini per riflessione.

\section{\1. Proprietà E natura Delle immagini tomografiche AD ULTRASUONI}

L'energia ultrasonica per formare l'immagine deve essere fornita da una sorgente esterna che può essere continua o impulsiva.

La creazione di immagini per riflessione impiega energia ultrasonica impulsiva.

La diagnostica ad ultrasuoni, sviluppata durante gli ultimi trenta/quaranta anni è usata di routine in molti settori quali: Ostetricia e Ginecologia, Oculistica, Internistica, Cardiologia e Neurologia.

Le caratteristiche peculiari degli ultrasuoni consistono nella possibilità che essi offrono di visualizzare le interfacce tra tessuti molli senza l'uso di mezzi di contrasto; nel consentire una metodica non invasiva né traumatica per il paziente; nel permettere di compiere misure di profondità e di eseguire stratigrafie su organi; nel fornire informazioni sul flusso ematico.

Con la parola ultrasuoni si indica la forma di energia meccanica, della stessa natura di quella emessa dalla bocca quando parliamo, a frequenze al di sopra della massima frequenza dei suoni udibili. La massima frequenza udibile dall'uomo è convenzionalmente di 16.000 oscillazioni al secondo, chiamate Herz $(\mathrm{Hz})$; in ecografia clinica per ora si usano frequenze da 1 a 20 milioni di $\mathrm{Hz}$.

Una caratteristica degli ultrasuoni è di propagarsi nei tessuti biologici molli ad una velocità sufficientemente bassa (circa 1500 metri al secondo) e molto prossima a quella di propagazione nell'acqua, cosicché sistemi elettronici possono essere impiegati per seguire l'evoluzione nel tempo dei segnali d'eco e fornire una rappresentazione che rende conto della morfologia di quanto gli ultrasuoni stanno illuminando all'interno del corpo umano.

Le onde ultrasoniche, a livelli di energia che non comportano danni né per il paziente né per l'operatore, vengono lanciati all'interno 
del corpo umano mediante un trasduttore piezoelettrico, detto anche sonda, cioè un elemento che eccitato elettricamente vibra e trasmette queste vibrazioni nel mezzo con il quale è in contatto.

Nella maggior parte delle applicazioni ecografiche gli ultrasuoni sono emessi sotto forma di impulsi, cioè pacchetti di vibrazioni; quando la vibrazione meccanica, lungo il cammino di propagazione, incontra un ostacolo (l'interfaccia tra un tessuto ed un altro, una discontinuità nel tessuto biologico, cavità ripiene di liquido, calcificazioni, ossa, bolle d'aria, corpi estranei) una parte dell'energia viene riflessa e una parte prosegue. Lo stesso trasduttore che ha emesso l'impulso ultrasonico, quando investito da vibrazioni meccaniche (segnali d'eco ultrasonici), fornisce ai suoi capi un segnale elettrico proporzionale a quello meccanico.

Il segnale elettrico viene trattato mediante dei circuiti elettronici e presentato molto frequentemente come segnale luminoso sullo schermo di un oscilloscopio; attraverso una correlazione che viene stabilita tra lo spostamento del punto luminoso sullo schermo e la distanza percorsa dagli ultrasuoni che si propagano all'interno dei tessuti, si crea una immagine della organizzazione delle strutture che sono state incontrate dagli ultrasuoni nella direzione di puntamento del trasduttore.

Principio analogo, cioè quello di rappresentare i segnali d'eco per creare immagini conformi del territorio esplorato dal sensore, viene utilizzato nelle apparecchiature sonar che impiegano onde meccaniche dello stesso tipo di quelle usate in ecografia clinica; le apparecchiature radar, anch'esse costituenti un sistema ecografico, si basano invece sull'impiego di onde elettromagnetiche.

Le immagini utilizzate in ecografia clinica possono essere del tipo tomografico (B-mode/Modo di Brillanza), oppure appaiono sotto forma di tracciati descrittivi di movimento di organi o parte di essi in funzione del tempo (TM-mode/modo di Movimento nel Tempo), lungo una singola direzione di puntamento della sonda; altri tracciati forniscono una rappresentazione della ampiezza dei segnali d'eco in funzione della distanza dalla sonda lungo una singola direzione di puntamento (A-Mode/Modo di Ampiezza); infine possono essere rappresentati sotto forma di tracciati gli andamenti temporali della ampiezza dei segnali Doppler (cioè di velocità di spostamento delle particelle del sangue) o di mappe cromatiche correlate al flusso ematico nelle cavità cardiache o nei vasi sanguigni. 
Nel seguito vengono riportati esempi di nuovi tipi di rappresentazione, da un lato legati al contenuto spettrale e locale del segnale ecografico e dall'altro ai vettori rappresentativi della velocità dovuta ad effetto Doppler di componenti del sangue in movimento.

\section{§2. SEgNALI A RADIOFREQUENZA}

Il segnale elettrico fornito da un traduttore ultrasonico in ricezione, è costituito da una oscillazione, la cosiddetta 'portante', centrata attorno alla frequenza centrale del trasduttore, con una modulazione di ampiezza e una modulazione di frequenza (Fig. 1). Questo segnale, che chiamiamo "segnale a radiofrequenza", può risentire di distorsioni non lineari dovute alla interazione tra ultrasuoni e materiale sotto indagine; questi effetti non lineari provocano nuovi contributi spettrali del segnale ricevuto rispetto a quello trasmesso.

\section{TRACCIATO A RADIOFREOUENZA DI UN SEGNALE ULTRASONICO RELATIVOA UN TESSUTO BIOLOGICO}

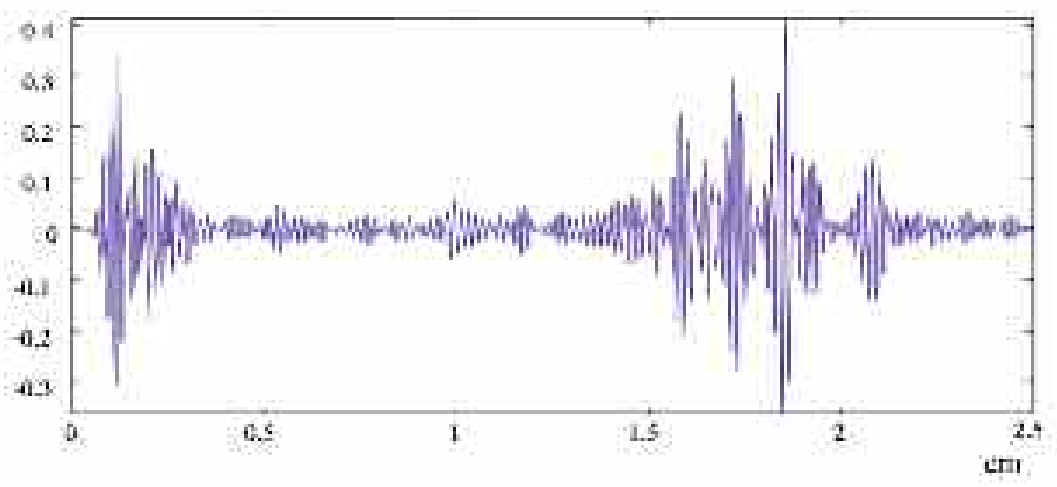

Fig. 1.

Segnale ad ultrasuoni a radiofrequenza generato dalla retrodiffusione dal parenchima di un tessuto biologico umano; sull'asse delle ascisse è riportata in centimetri la profondità nel tessuto. 
Pertanto, se la forma del segnale a radiofrequenza è persa perché si opera l'estrazione di un inviluppo, rappresentato in Fig. 2 dalla linea più grossa in rosso che appunto inviluppa il segnale, non si possono utilizzare tutte le informazioni che il segnale riporta per effetto dell'interazione di tipo lineare e non lineare tra ultrasuoni e mezzo studiato. Per i tessuti biologici le interazioni lineari da un lato provocano tutte quelle modificazioni dello spettro del segnale trasmesso dovute ad una attenuazione selettiva in frequenza (Fig. 3), dipendente anche dalla profondità; dall'altro, quando le dimensioni medie degli elementi strutturali del tessuto biologico sono confrontabili con la lunghezza d'onda ultrasonica, la interferenza di questi segnali, retrodiffusi dalla varie discontinuità, provoca una modificazione sullo spettro di ampiezza (Fig. 4) e di fase del segnale; queste variazioni non sono più rilevabili se si utilizza il solo inviluppo del segnale e non il segnale completo a radiofrequenza. L'interferenza nel dominio del tempo è responsabile delle modificazioni in ampiezza nel dominio delle frequenze e dell'andamento della fase modificato rispetto al segnale trasmesso.

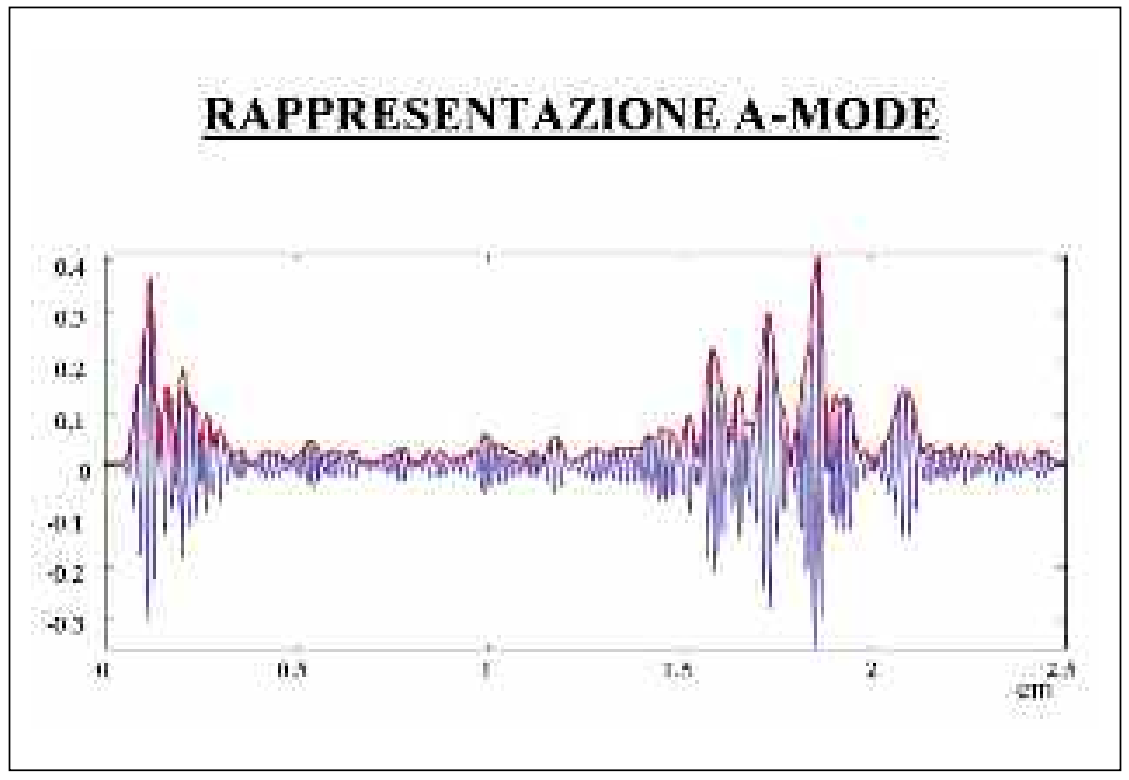

Fig. 2.

Tracciato a radiofrequenza descritto in Fig. 1 con sovrapposto l'andamento dell'inviluppo; sull'asse delle ascisse è riportata in centimetri la profondità del tessuto. 


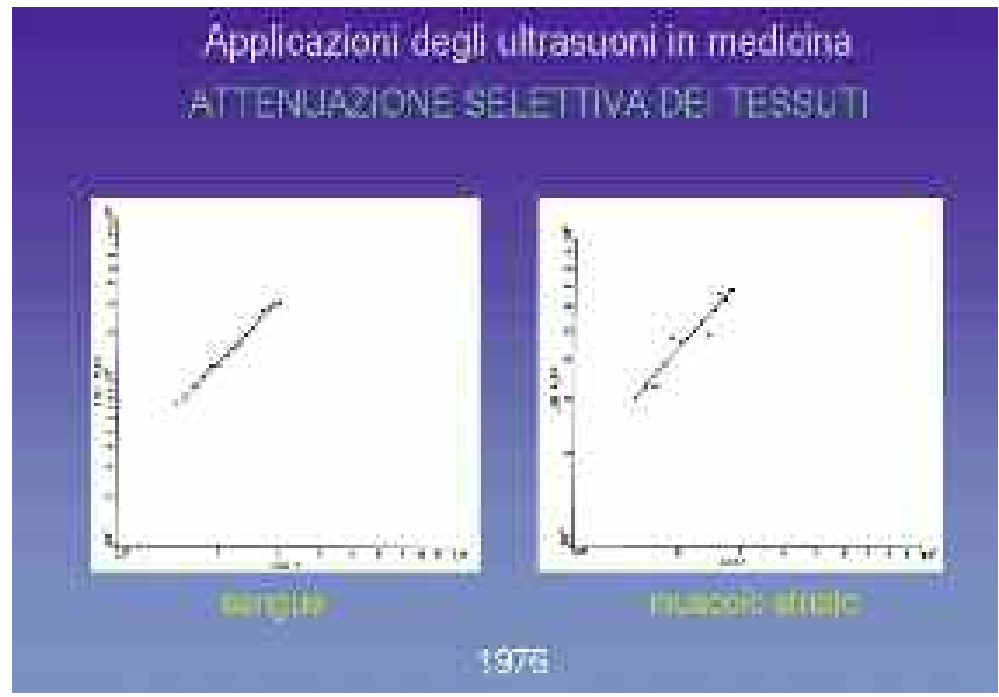

Fig. 3.

Andamento dell'attenuazione in funzione della frequenza per sangue umano e per muscolo striato.

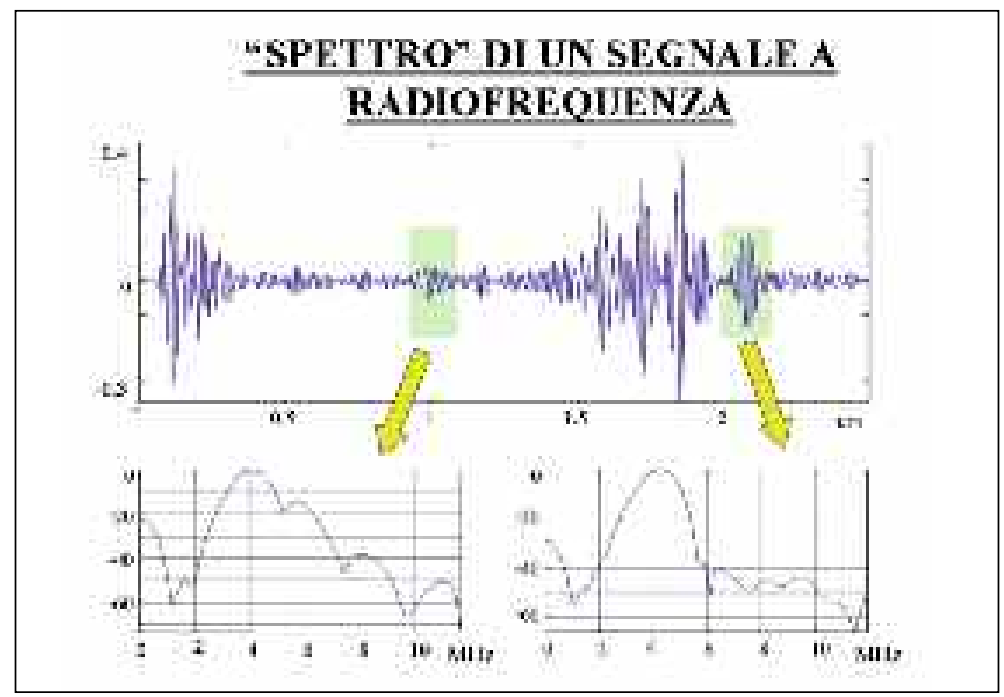

Fig. 4.

Tracciato a radiofrequenza con andamento dello spettro in due diverse porzioni di tempo, corrispondenti a due diverse profondità nel tessuto biologico. 
Analogamente, il preservare l'intera banda del segnale a radiofrequenza è essenziale per raccogliere l'informazione derivante dalle interazioni non lineari; le interazioni non lineari derivano dalla dipendenza delle proprietà locali del tessuto biologico quali: impedenza acustica, legata alla densità, che a sua volta dipende dalla intensità della stessa onda ultrasonica incidente.

Quando si usino mezzi di contrasto per gli ultrasuoni, costituiti da microbolle di gas, di diametro di alcuni micron, iniettati all'interno del corpo umano e trasportati dal sangue lungo i grandi e i piccoli vasi, gli effetti di interazione non lineare tra pressione acustica e dimensione delle bolle, creano nel segnale retrodiffuso non solo spettri armonici ma anche spettri subarmonici. In Fig. 5 è mostrato l'andamento, nel dominio del tempo e nel dominio della frequenza, del segnale retrodiffuso da

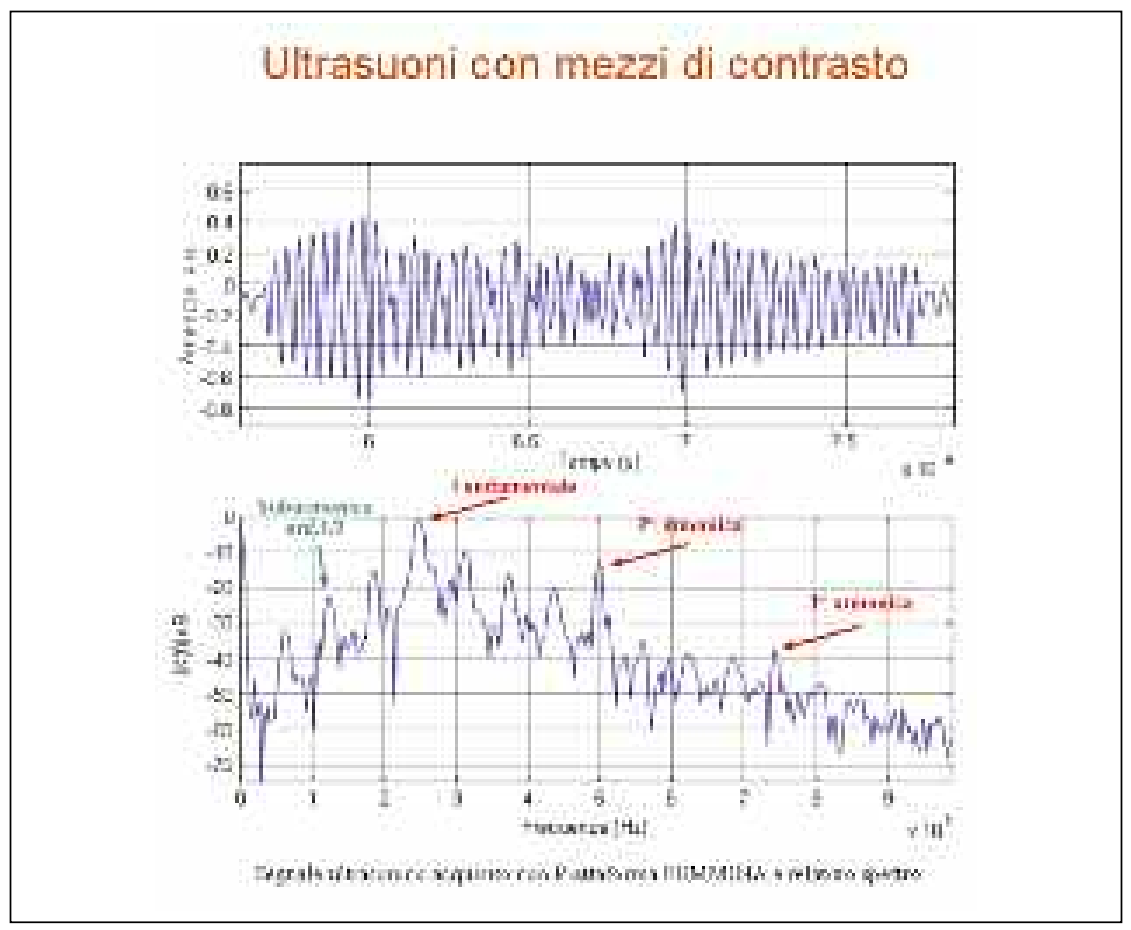

Fig. 5.

Andamento, nel dominio del tempo e nel dominio della frequenza, del segnale retrodiffuso da un mezzo di contrasto per ultrasuoni; nell'andamento spettrale dell'ampiezza si nota la presenza di componenti fondamentali, armoniche e subarmoniche. 
un mezzo di contrasto per ultrasuoni; nell'andamento spettrale dell'ampiezza si nota la presenza di componenti fondamentali, armoniche e subarmoniche. Nella Fig. 6 sono mostrate mappe spettrali che abbiamo rilevato su mezzi di contrasto al variare del tempo; si nota una non contemporaneità nella presenza degli spettri armonici e subarmonici che si può interpretare come determinata dalla evoluzione del tipo di interazione tra ultrasuoni e mezzi di contrasto nei tempi di arrivo dell'eccitazione degli ultrasuoni e di susseguente ritorno alla situazione di riposo.

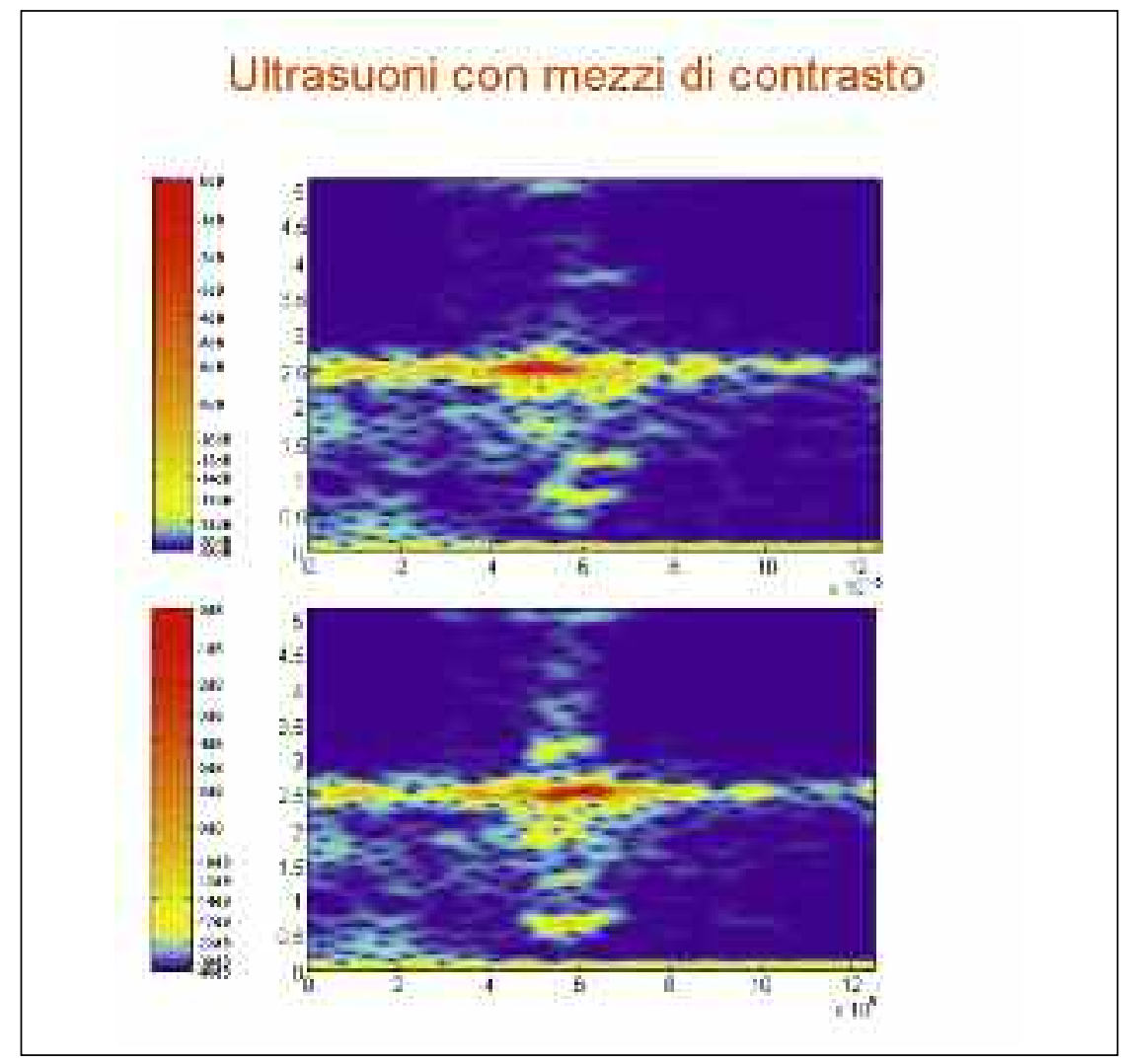

Fig. 6.

Sono mostrate mappe spettrali che abbiamo rilevato su mezzi di contrasto al variare del tempo; si nota una non contemporaneità sulla presenza degli spettri armonici e subarmonici che si può interpretare come determinata dalla evoluzione del tipo di interazione tra ultrasuoni e mezzi di contrasto nei tempi di arrivo dell'eccitazione degli ultrasuoni e di susseguente ritorno alla situazione di riposo. 


\section{\$2.1 Differenziazione di tessuti biologici mediante elaborazione del segnale ecografico a radiorequenza}

Basandosi sulla elaborazione dei segnali ecografici a radiofrequenza si sono effettuati esperimenti di differenziazione di tessuti biologici sia "in vitro" che "in vivo" utilizzando una nuova piattaforma di elaborazione veloce del segnale a radiofrequenza; ad esso è stato dato il nome di 'FEMMINA', come acronimo di Fast Echographic Multiparameter Multi Image Novel Apparatus.

\section{\$3. SISTEMI ELETTRONICI PER ECOGRAFI DI NUOVA GENERAZIONE CHE UTILIZZANO IL SEGNALE A RADIOFREQUENZA}

'FEMMINA' è una piattaforma hardware-software dedicata all'elaborazione di segnali ed immagini in grado di consentire anche la visualizzazione veloce dei dati. Il suo campo elettivo di applicazione è quello biomedico, dove l'elaborazione veloce risulta necessaria al fine di riuscire a valutare le prestazioni dei diversi metodi di indagine in sperimentazione clinica ma l'architettura base può essere utilizzata anche per controlli non distruttivi ad ultrasuoni o per acquisire ed elaborare segnali provenienti da sensori ottici. L'idea alla base di questa piattaforma è la completa integrazione di una sezione hardware per l'elaborazione in tempo reale con l'architettura di un Personal Computer (Fig. 7). Questo permette di ottimizzare la distribuzione dell'onere di calcolo fra hardware e software al fine di ottenere una produzione continua di immagini anche nei casi in cui sia richiesta una pesante elaborazione. Il software a bordo del PC ha il compito di configurare ed assumere il controllo della sezione hardware, di effettuare ulteriori elaborazioni sui segnali ecografici acquisiti, e di visualizzare le immagini risultanti; il PC agisce anche da interfaccia utente interattiva per l'intero sistema.

L'architettura del sistema è modulare, espandibile. Essa è totalmente programmabile e, a seconda dell'applicazione, può essere riconfigurata in linea in base ai parametri ultrasonici che devono essere stimati di volta in volta. La modularità e l'espandibilità, sia per quanto riguarda la sezione hardware sia per quella software, danno la possibilità di sostituire o aggiungere ulteriori schede digitali dedicate all'elaborazione in tempo reale o ulteriori moduli software finalizzati a di- 
versi metodi di indagine. Questi due aspetti, uniti alla programmabilità della piattaforma, permettono di implementare e sperimentare con estrema facilità nuovi algoritmi per l'elaborazione del segnale.

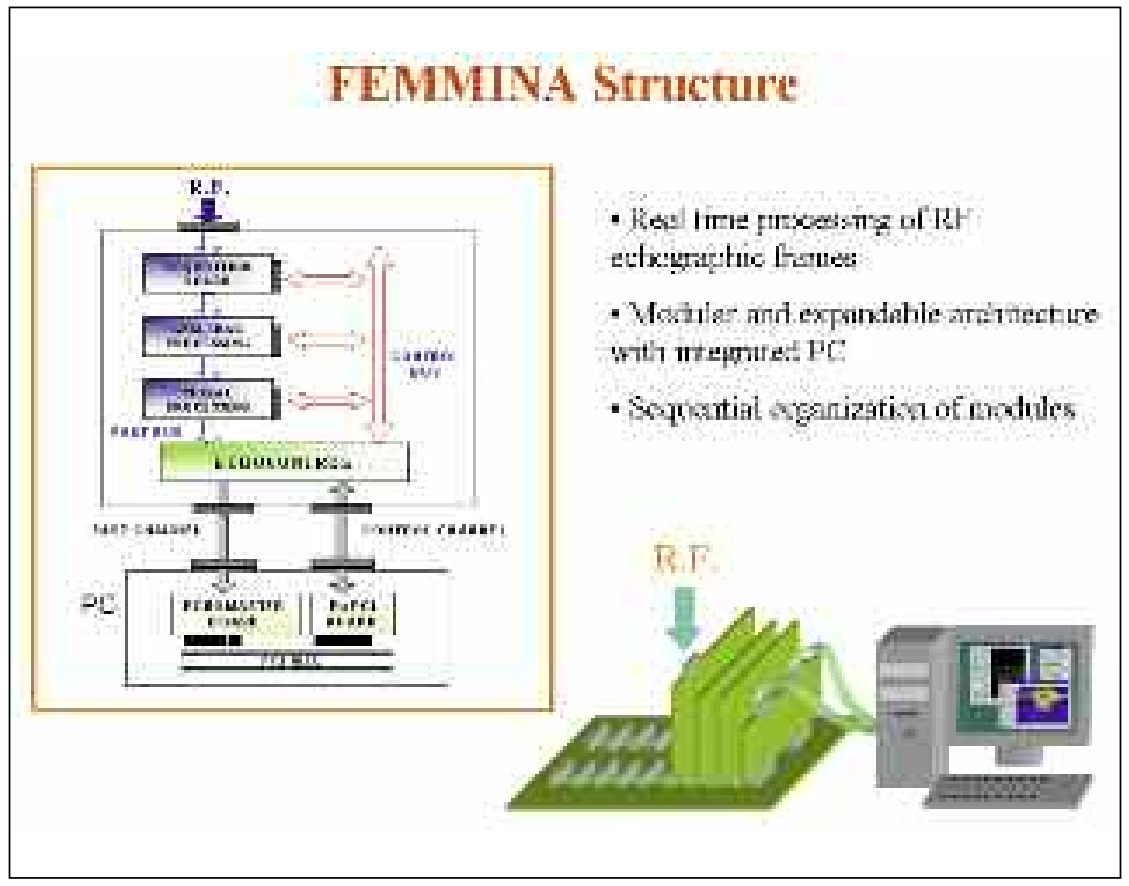

Fig. 7.

Struttura della piattaforma 'FEMMINA'.

I risultati delle elaborazioni per la estrazione di vari parametri possono essere rappresentati contemporaneamente sullo schermo, così come le strutture e le caratteristiche delle elaborazioni eseguite (Fig. 8).

È stato sviluppato un nuovo metodo di differenziazione di tessuti biologici. [1] Alcuni parametri descritti dall'andamento dello spettro locale, cioè riferito ad una piccola porzione del tessuto esaminato, si ottengono dalle mappe spettrali nelle quali si possono osservare distinte le immagini di tessuti tra loro diversi o parti non omogenee affette da patologie. I parametri dello spettro locale sono correlabili alla microstruttura del tessuto indagato con ultrasuoni e sono presentati su mappe riferite o sovrapposte alla porzione di tessuto studiato, in abbinamento a tomografie ecografiche simili a quelle ottenute con gli ecografi tradizionali. 


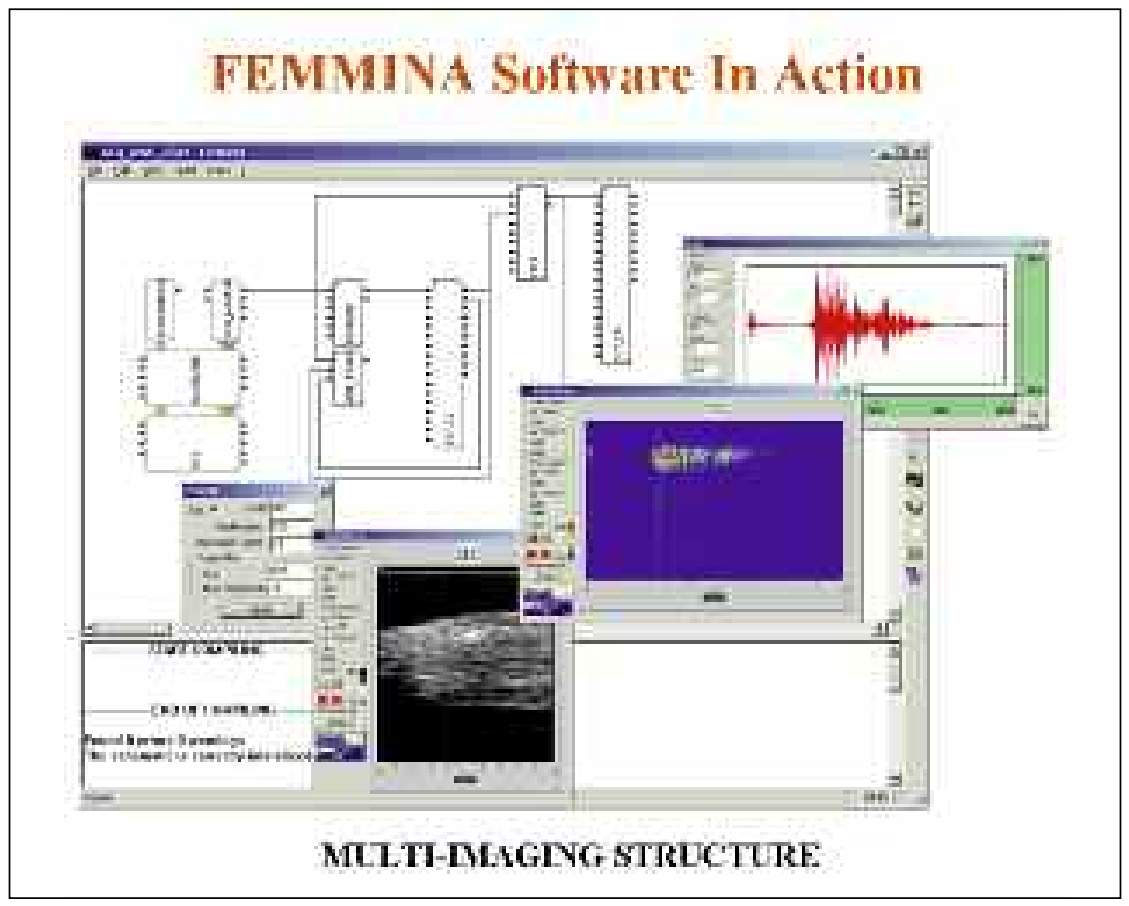

Fig. 8

Esempio di rappresentazione multipla di parametri, tracciati, immagini e struttura di scelta della elaborazione del segnale.

In Fig. 9 è riportato il risultato ottenuto sulla individuazione di sangue non in movimento per una prova "in vitro"; in Fig. 10 è rappresentato il risultato ottenuto sulla carotide di un volontario. In entrambi i casi il sangue è stato evidenziato mediante la rappresentazione di parametri legati alla forma dello spettro del segnale ecografico a radiofrequenza. Il metodo è stato esteso allo studio di prostata "in vitro" e "in vivo": è stato verificato con successo che i risultati ottenuti con gli ultrasuoni coincidono con le osservazioni compiute dagli anatomopatologi che indipendentemente hanno studiato i reperti nelle stesse sezioni che erano state usate per le ecotomografie. Sono stati individuate porzioni della ghiandola affette da carcinoma e, in altri casi, porzioni di tessuto modificato per adenoma; sono stati esaminati finora (giugno 2002) 42 casi, con corrispondenza totale tra $i$ dati emersi dalla nostra indagine e quelli indipendentemente trovati attraverso l'esame istologico. 


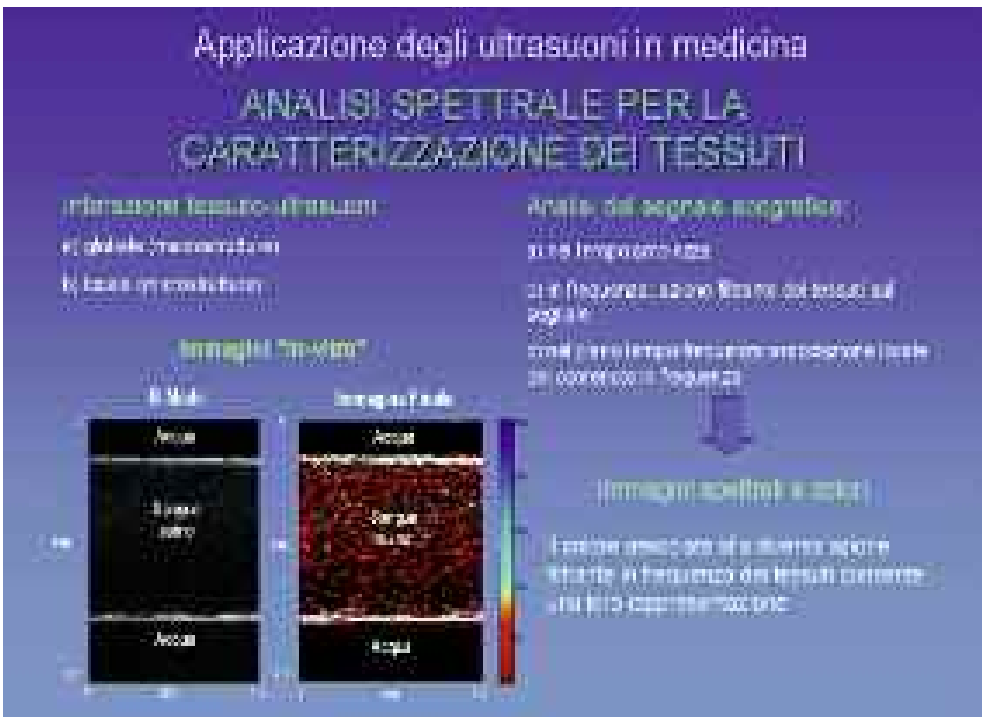

Fig. 9.

Risultato ottenuto sulla individuazione di sangue non in movimento per una prova "in vitro".

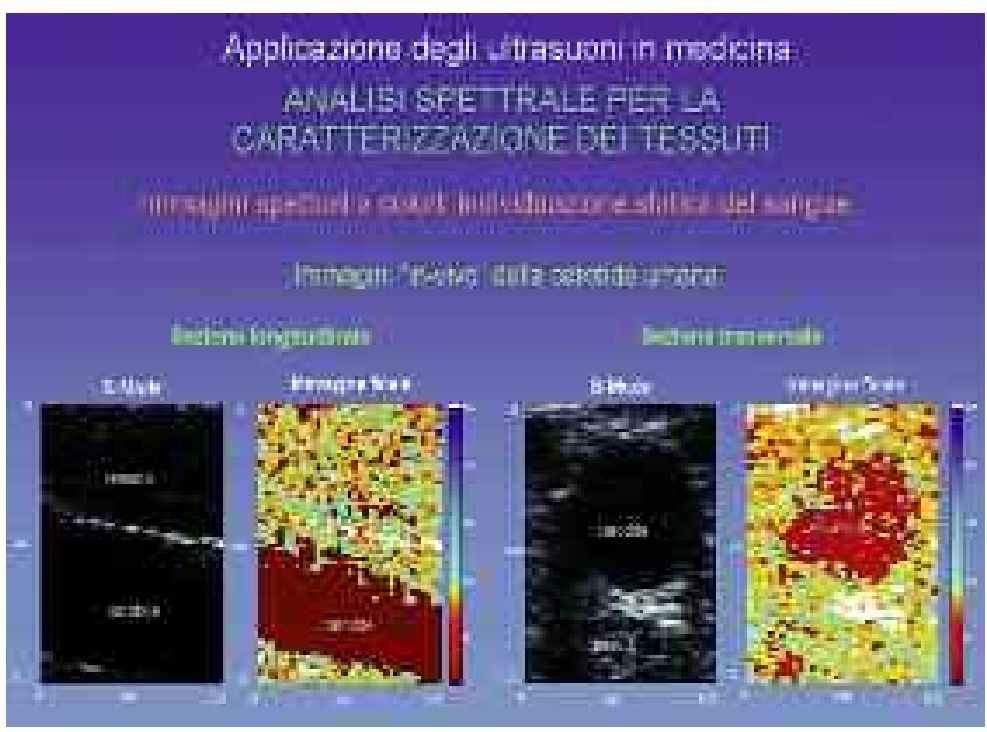

Fig. 10.

Rappresentazione del risultato di rivelazione del sangue ottenuto sulla carotide di un volontario. 
Sono state eseguite sezioni ecografiche ed esami istologici su sezioni della ghiandola separate da un millimetro: in Fig. 11 e 12 sono mostrate le immagini pertinenti.

La possibilità di estrarre caratteristiche differenziali dei tessuti biologici ha suggerito lo sviluppo di microsonde ad ultrasuoni ricetrasmittenti a larga banda.

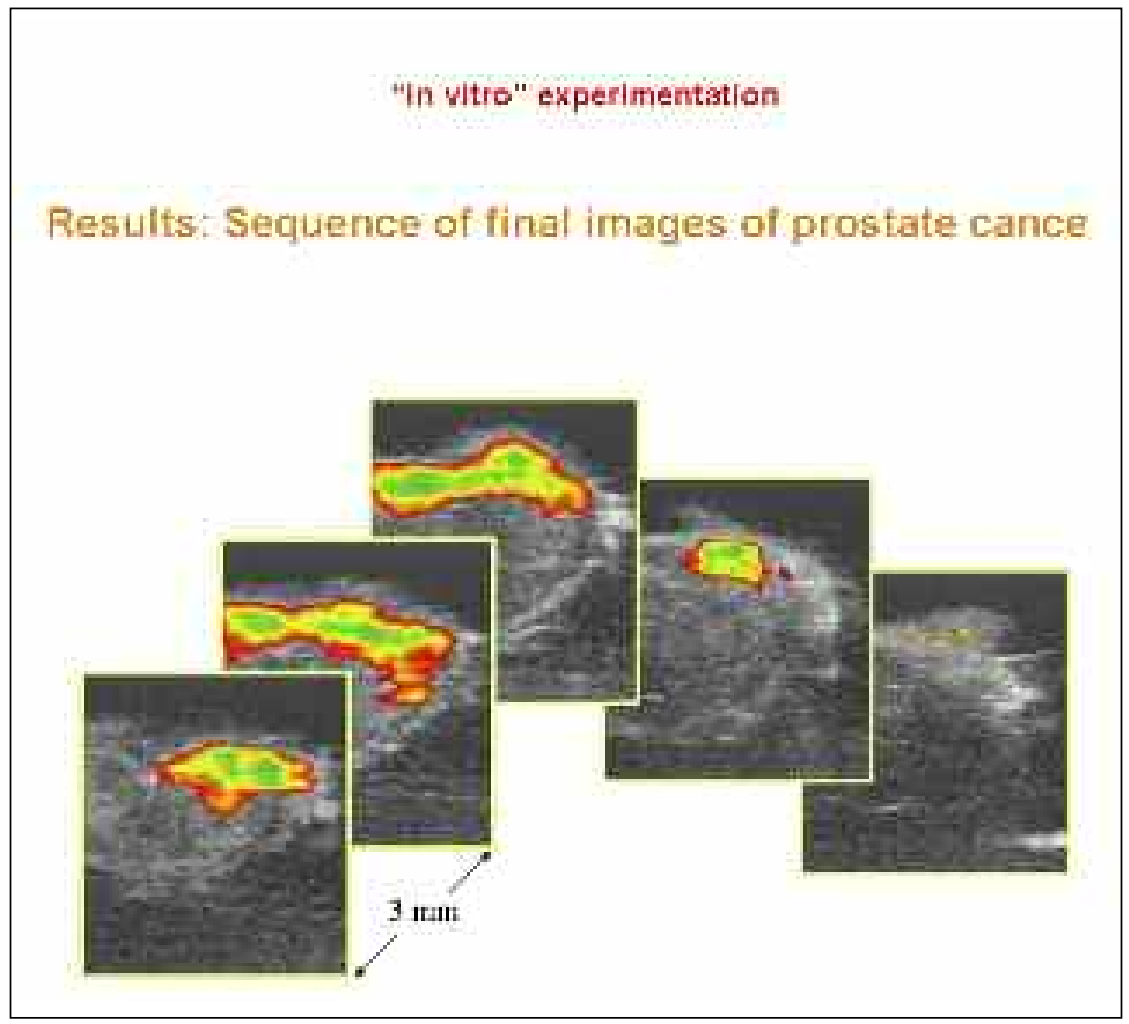

Fig. 11.

Immagini relative alla sezione anatomica e alla immagine ecotomografica elaborata. Viene mostrata la sequenza di sezioni anatomiche distanti reciprocamente $1 \mathrm{~mm}$ lungo piani coincidenti con quelli utilizzati per l'acquisizione dei segnali ecografici. 


\section{METODO DI SCANSIONE DELLE IMMAGINI}

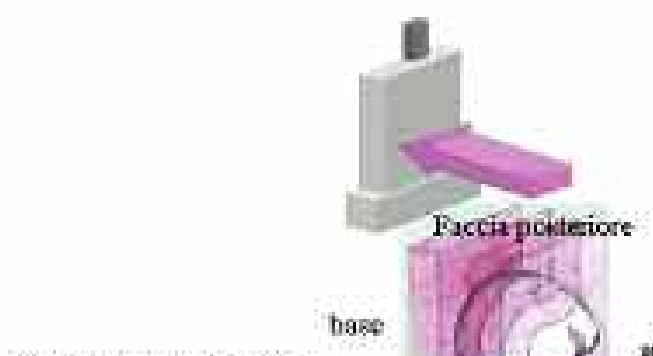

Serione anatomica

Faceta postcriore

\section{Faccia anteriute} Immagine elahorata

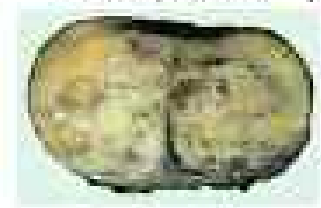

Fatcia interioce

spice

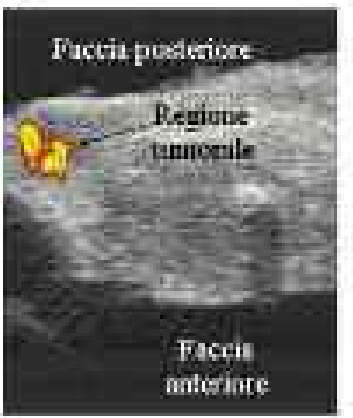

Fig. 12.

Rappresentazioni analoghe a quelle mostrate in Fig. 11.

\section{\4. MICROSONDE AD ULTRASUONI PER BIOPSIA VIRTUALE}

Come risultato di molte indagini diagnostiche, dalla palpazione alla risonanza nucleare, alla radiografia (mammografia, tomografia assiale computerizzata, radiografia tradizionale) alla ecografia, si rilevano masse anche di piccole dimensioni, in forma di lesioni focali, delle quali è necessario stabilire l'origine, la natura. La metodica che si vuole sviluppare può portare un contributo di caratterizzazione tessutale fondato su una differenziazione dei tessuti biologici molli sulla base della organizzazione strutturale tipica. Gli ultrasuoni, essendo onde mecca- 
niche, interagiscono con il mezzo nel quale si propagano, in maniera intima e fortemente influenzata dalle caratteristiche meccaniche. Nel caso del tessuto biologico, tali caratteristiche sono la elasticità e la densità locale e globale e le modalità di microagglomerazione e di macroagglomerazione. Inoltre la presenza di microvasi in maggiore o minore quantità come accade tipicamente per molti tessuti tumorali, modifica la organizzazione del segnale ultrasonico che, dopo aver interagito con tali porzioni di tessuto, ritorna al sistema ricevente.

Più largo è lo spettro del segnale ultrasonico trasmesso e ricevuto, maggiore è la ricchezza di componenti spettrali e quindi di potenzialità di studio delle modificazioni indotte dal mezzo di propagazione. Nel dominio del tempo il disporre di segnali a larga banda significa piccole durate temporali e di conseguenza alta risoluzione spaziale che comporta la possibilità di risolvere strutture piccole, tipiche della organizzazione dei tessuti biologici. Un esempio numerico: un segnale con una banda di $50 \mathrm{MHz}$ che si propaga nel tessuto biologico molle può fornire una risoluzione teorica in distanza, di circa quindici micrometri; tale dimensione è comparabile con quelle di una cellula e quindi un tale segnale è in grado di essere modificato sensibilmente nella sua propagazione, in base alla organizzazione degli agglomerati di cellule. D'altra parte gli ultrasuoni sono assorbiti dai tessuti biologici molli maggiormente all'aumentare della frequenza, pertanto non sarebbe possibile lo studio proposto impiegando le tradizionali tecniche di esame ecografico di un paziente, cioè con un approccio mediante sonde esterne a frequenza relativamente bassa (alcuni megaherz), appoggiate alla pelle del paziente, in generale lontane dalle lesioni focali. Le alte frequenze consentite dalle microsonde che abbiamo proposto, da un lato aprono la possibilità di studiare caratteristiche di microstruttura dei tessuti biologici dall'altro, dato il forte aumento della attenuazione degli ultrasuoni all'aumentare della frequenza, potranno essere esplorati tessuti per profondità di pochi millimetri: le lesioni focali saranno investigate con le microsonde poste in vicinanza di esse.

La ricerca svolta dal gruppo e in corso di completamento, ha portato a realizzare versioni preprototipali di microsonde riceventi e trasmittenti in fibra ottica in grado di essere portate in prossimità della lesione focale o all'interno di sottili aghi oppure all'interno di endoscopi o cateteri (Fig. 13), in dipendenza dalla situazione. Le sonde sono caratterizzate da dimensioni molto piccole e da una banda in trasmissione e in ricezione molto ampia, fino a $70 \mathrm{MHz}$. Il principio sul quale si basa il di- 
spositivo trasmittente è la conversione di un impulso laser in un impulso a ultrasuoni avente larghezza di banda imposta dalla durata dell'impulso laser; l'impulso laser viene trasmesso lungo una fibra ottica sulla cui estremità è realizzato il convertitore optoacustico che funziona da elemento trasmittente (Fig. 14): il sistema è stato da noi brevettato su piano mondiale. Il principio sul quale si basa il ricevitore è una conversione acusto-ottica: l'onda di pressione degli ultrasuoni modifica la riflessione dell'estremità di una fibra ottica che canalizza una sorgente luminosa costituita da un laser di piccola potenza (Fig. 15); la modulazione dell'intensità della luce riflessa è linearmente legata alla intensità degli ultrasuoni che colpiscono il dispositivo che nel nostro caso è il risultato del segnale eco riflesso e retrodiffuso dal tessuto illuminato $[2,3]$.

\section{Ultrasonic transducers based on fiber optics}

\section{- High frequency and wide baud \\ - Miniatarixation \\ Micrescopic tissue characterikation \\ Minimal invasive intervention}

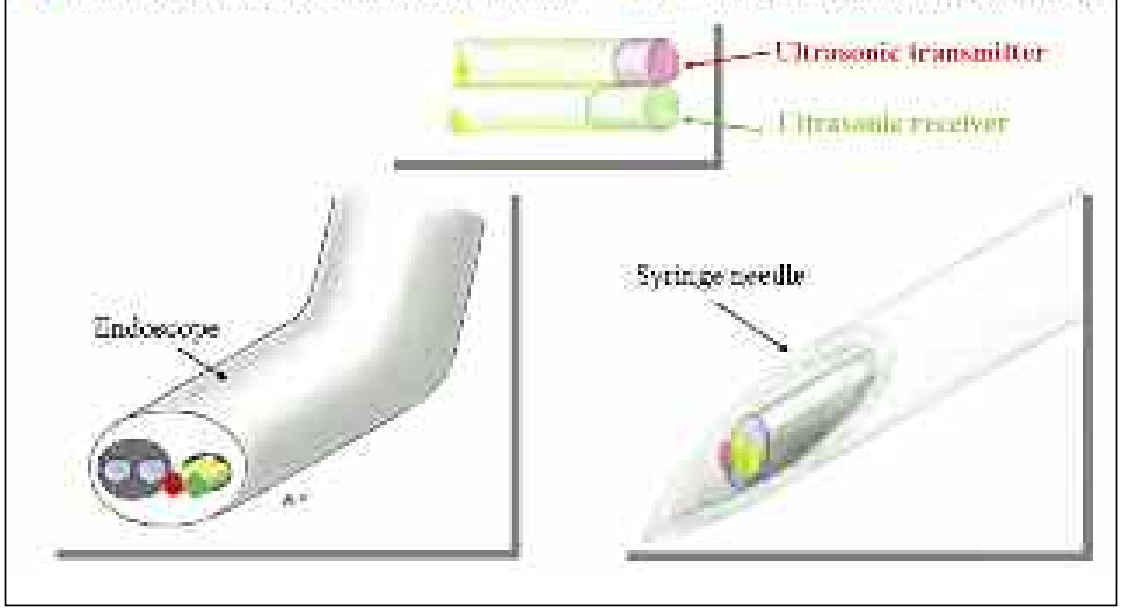

Fig. 13.

Microsonda per indagini su lesioni focali, mediante endoscopio (figura in basso a sinistra), oppure mediante ago (figura in basso a destra). Nella parte superiore sono rappresentate le due fibre opportunamente terminate, una destinata alla generazione di ultrasuoni e l'altra alla ricezione. 
I risultati ottenuti fanno prevedere la possibilità di realizzare un trasduttore completo ad ultrasuoni costituito da due fibre ottiche. Le due fibre possono essere montate adiacenti e vengono a costituire il collegamento tra l'apparecchiatura trasmittente ricevente e la porzione sotto esame. Occorre inoltre osservare che non si ha, con queste sonde, contatto galvanico tra strumentazione e pazienti e quindi senza pericolo di portare correnti elettriche all'interno del corpo umano.

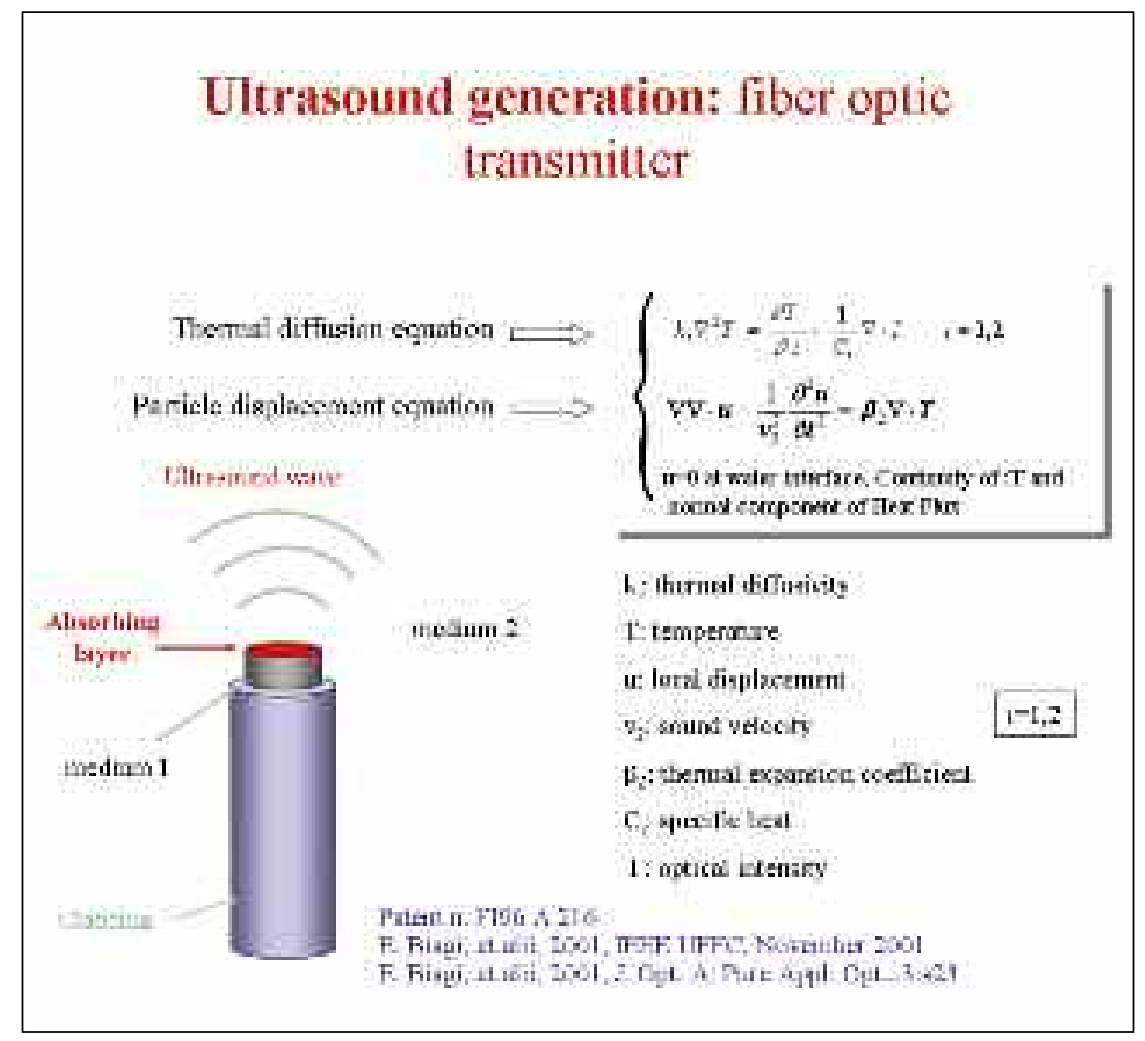

Fig. 14.

Microsonda per la generazione di ultrasuoni: rappresentazione schematica. 


\section{Ultrasound detection: interferometric method}

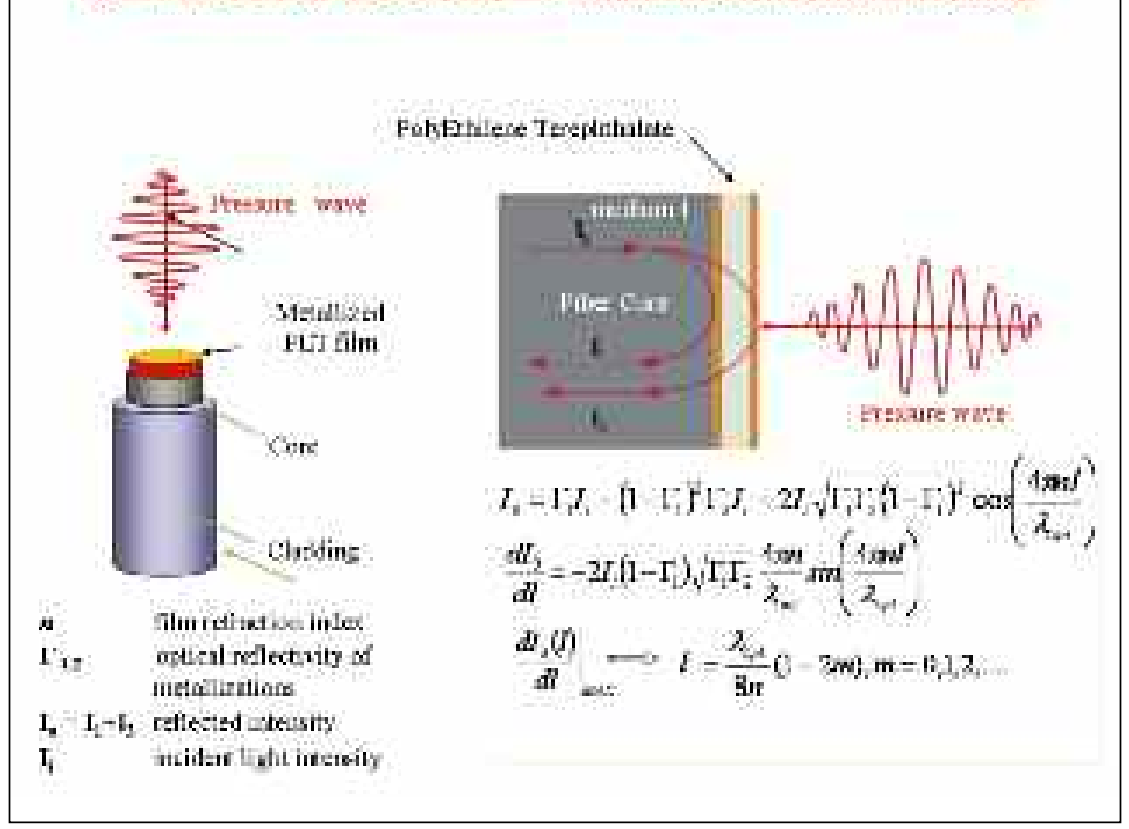

Fig. 15.

Microsonda per la ricezione degli ultrasuoni: rappresentazione schematica

\5. SISTEMA TRIDIMENSIONALE DI ACQUISIZIONE E PRESENTAZIONE DI DATI

Come in altre modalità di creazione di immagini in medicina, la Tomografia Assiale Computerizzata e la Risonanza Magnetica Nucleare, gli Ultrasuoni hanno la possibilità teorica di generare immagini tridimensionali. La visualizzazione degli organi interni in tre dimensioni può rivelare nuove informazioni che possono aiutare i medici nella diagnosi, nel calcolo dei volumi e nella pianificazione chirurgica. Nel 1988 [6] si è sviluppato un sistema brevettato su base mondiale che ha permesso di ottenere immagini tridimensionali del cuore mediante ultrasuoni. Il sistema è costituito da una sonda, di dimensioni 
confrontabili con quelle delle sonde bidimensionali classiche, che è strutturata in modo tale da raccogliere dati con riferimenti spaziali noti in un intero volume; la sonda compie scansioni settoriali e dopo ogni scansione ruota attorno al suo asse, con la sequenza come mostrato nella Fig. 16. Il sistema consente di ottenere i dati ecografici da un volume di forma tronco-conica, oggetto dello studio. Dalla quantità di dati ottenuta è possibile ottenere sezioni in qualunque piano del cono anche per organi in movimento; per il cuore si ottiene la possibilità da parte del medico di studiare piani valvolari non altrimenti accessibili con la ecografia classica oppure delle sezioni del cuore in piani non direttamente accessibili e confrontare il movimento della sezione del cuore per diagnosticare eventuali discinesie; altre applicazioni della tridimensionalità sono in fase di sviluppo anche per altri organi.

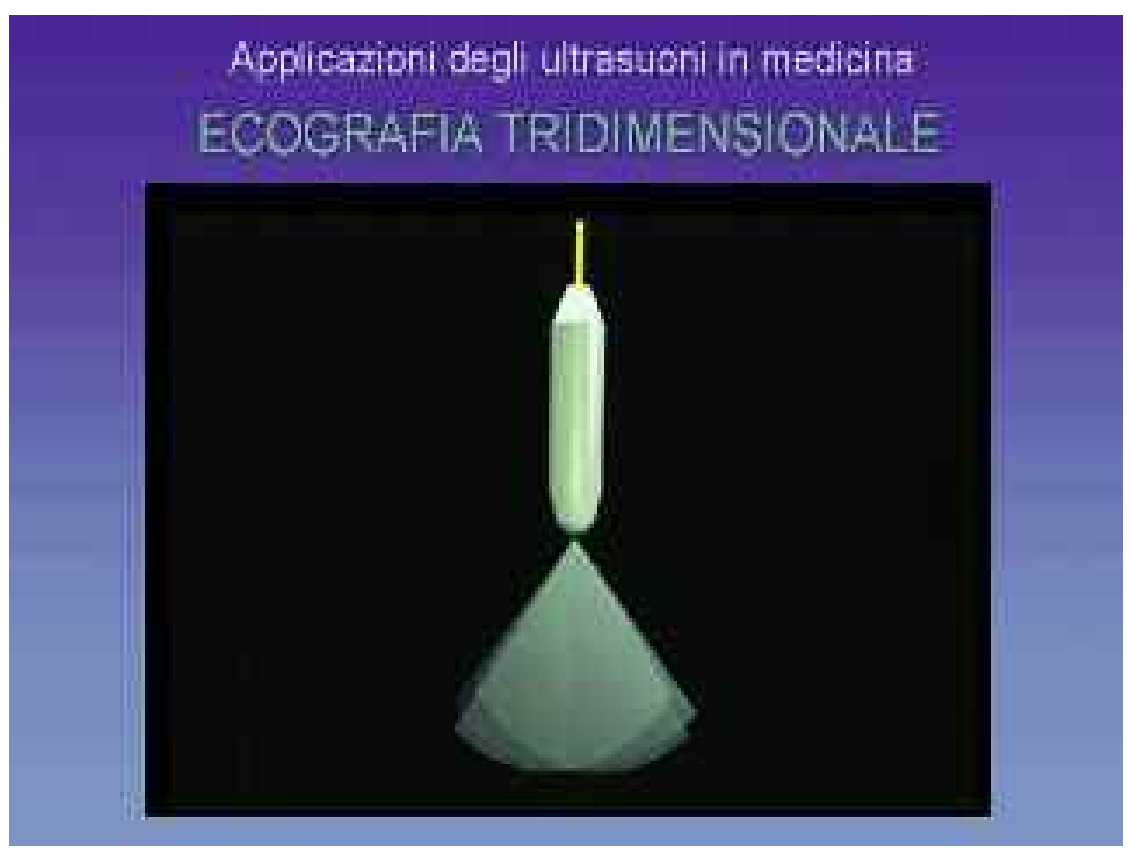

Fig. 16.

Sonda per acquisizione di dati ecografici tridimensionali. 


\section{$\$$ 6. DOPPLER VETTORIALE}

Il Doppler vettoriale sul quale si è lavorato fin dal 1994 è ottenuto mediante la rilevazione delle tre componenti del vettore velocità rilevata mediante l'effetto Doppler sugli ultrasuoni trasmessi [4,5]. La struttura, come mostrato in Fig. 17, in una forma di prototipo sperimentale realizzato, è costituita da un trasduttore centrale che emette, e tre trasduttori riceventi ad angolazione conosciuta rispetto a quello trasmittente. In questo modo possono essere ricostruiti i segnali raccolti dai tre trasduttori riceventi con la loro orientazione angolare, nota a priori. Inizialmente sono stati svolti esperimenti per verificare la fattibilità del sistema ed in particolare si è eseguita la prova su un disco rotante con una superficie diffondente per gli ultrasuoni immerso in acqua.

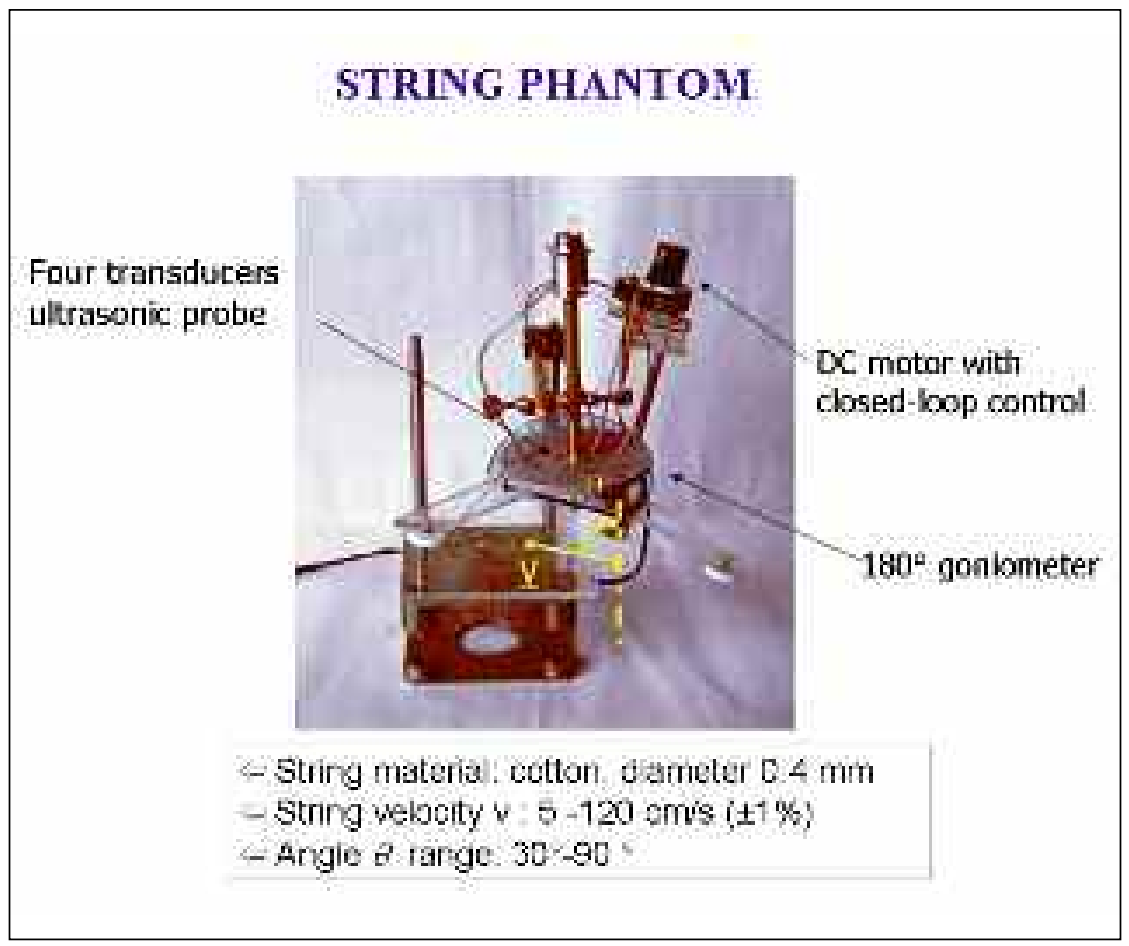

Fig. 17.

Struttura sperimentale per acquisire segnali Doppler vettoriali costituita da tre trasduttori riceventi e un trasduttore trasmittente 
Come appare in Fig. 18, si è ottenuta una immagine nella quale sono presentate le areole dell'immagine ottenuta, ognuna caratterizzata da un un versore che ha una inclinazione omogenea con quella che è la direzione del vettore vero: in codice cromatico è rappresentato il valore del modulo della velocità locale e, come era prevedibile, una metà del disco è caratterizzata dalla velocità negativa e l'altra metà del disco è rappresentata dalla velocità positiva; cioè specificamente in allontanamento e in avvicinamento rispetto alla sorgente ultrasonica ricetrasmittente. Sono state fatte esperienze anche su oggetti campioni ottenuti con fili messi in movimento nell'acqua e a valle di queste sperimentazioni è stato esteso il metodo ad un trasduttore di tipo elettronico a cortina fasata della Esaote Biomedica; il sistema a cortina lineare elettronica è stato alimentato mediante schede sviluppate appositamente.

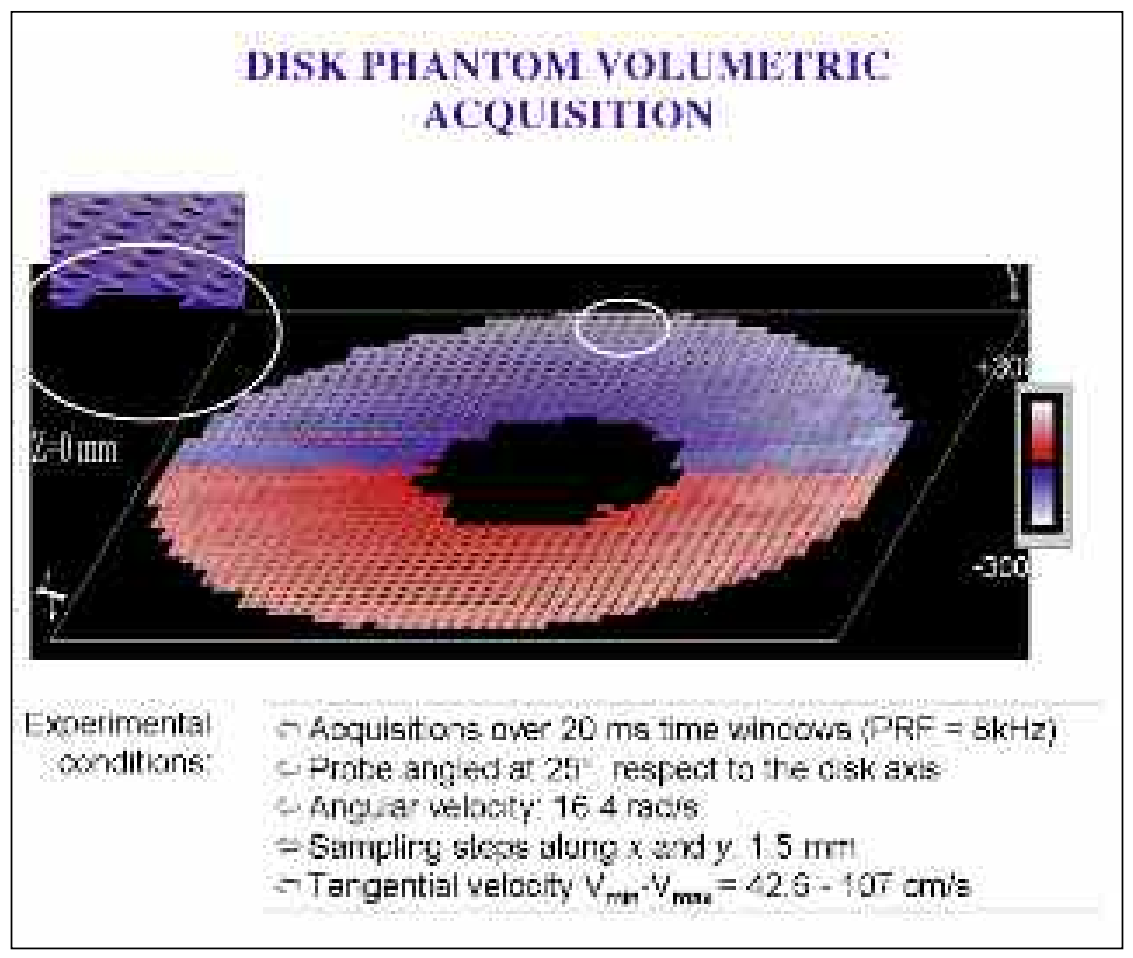

Fig. 18.

Mappa ottenuta mediante il sistema Doppler tridimensionale in prove di laboratorio. 
La gestione del trasduttore avviene come mostrato in Fig. 19; la scansione viene eseguita per scorrimento laterale di una coppia di gruppi di trasduttori riceventi e per traslazione del gruppo trasmittente situato sulla mezzeria rispetto ai due riceventi. In tal modo viene esplorata un'area piana ottenendo le informazioni vettoriali delle due componenti nel piano; anche in questo caso essendo noti gli angoli tra il trasduttore trasmittente e quelli riceventi. È allo studio un sistema di cortina realizzato a forma di $\mathrm{Y}$ in modo tale da ottenere di nuovo la informazione del vettore nello spazio nelle tre dimensioni. Abbiamo eseguito con la struttura bidimensionale così realizzata delle prove anche "in vivo" e i risultati sono mostrati nella Fig. 19, riferentesi a tre voxel, all'interno di una porzione di carotide di un giovane volontario.

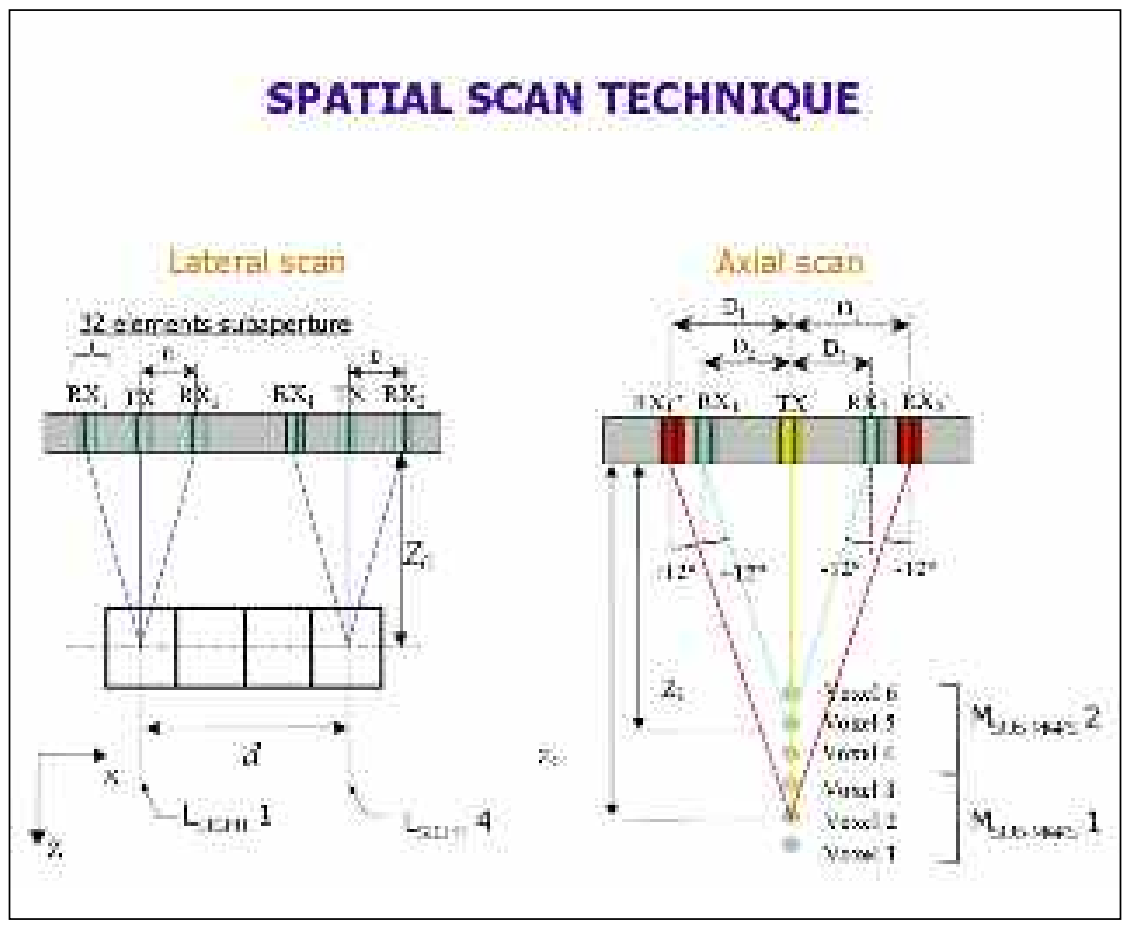

Fig. 19.

Sonda lineare a cortina fasata utilizzata per rilevare il vettore Doppler nel piano di scansione. I due gruppi di elementi laterali riceventi scorrono lungo la cortina con comando elettronico, analogamente il gruppo centrale trasmittente scorre mantenendosi sulla mezzeria dei due gruppi riceventi. 
Nella Fig. 20 è mostrato il sistema applicato ad una struttura di oggetto campione ottenuta mediante la fabbricazione di un tubo di flusso con una immissione laterale. Si nota che la immissione di flusso dal tubo laterale di sezione ridotta crea una turbolenza con una inversione di velocità, mostrata in blu sul resto della figura in rosso, ovvero velocità in avvicinamento rispetto al sistema dei trasduttori ultrasonici.

Uno dei problemi più importanti che si ha nel visualizzare l'effetto Doppler come valore di velocità e di sua orientazione, è la difficoltà della rappresentazione dei dati. La rappresentazione di un campo vettoriale tridimensionale è un problema non ancora risolto, già presente nello studio della velocità delle particelle dei plasma. Due metodi che abbiamo sviluppato sono mostrati nelle Fig. 18 e Fig. 21 in quest'ultima l'effetto Doppler vettoriale, o tridimensionale, viene mostrato secondo una rappresentazione polare.

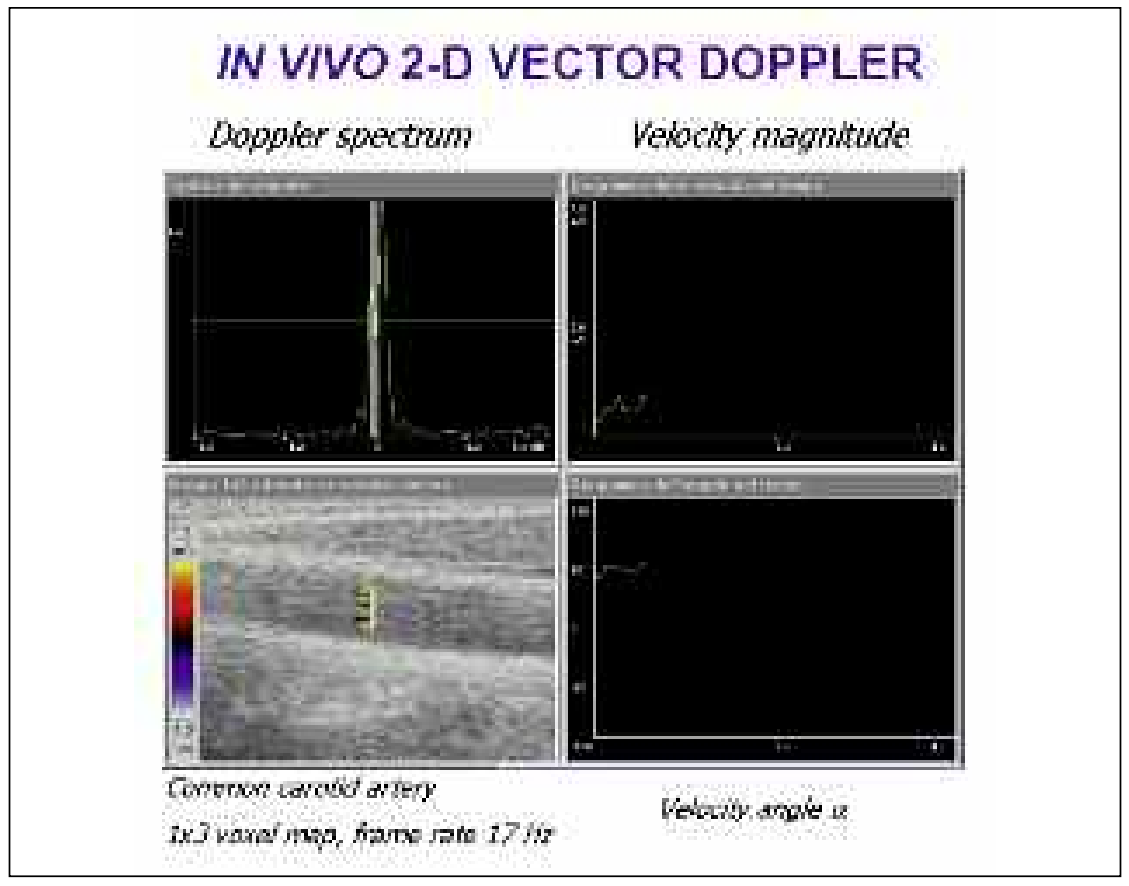

Fig. 20.

Immagine ottenuta dalla rilevazione del Doppler vettoriale in arteria carotidea (in basso a sinistra) e tracciati delle grandezze caratteristiche. Le immagini e le informazioni Doppler sono state rilevate mediante la sonda schematicamente mostrata nella figura 19. 


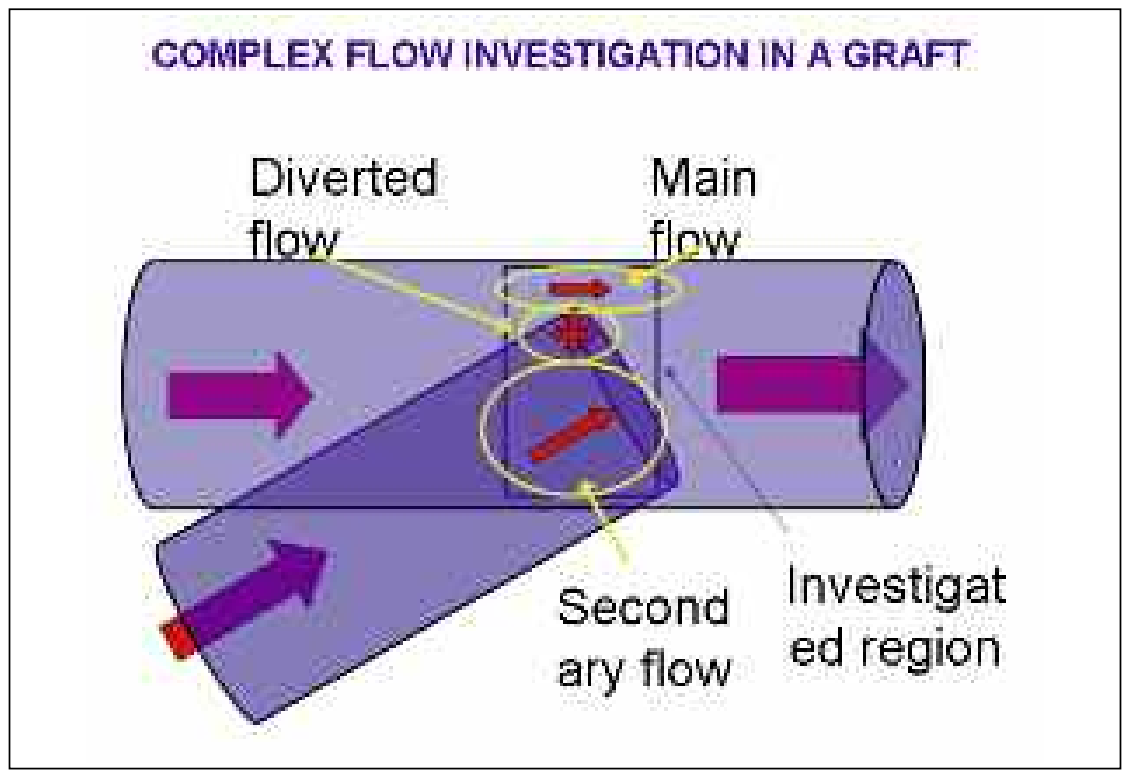

Fig. 21.

Struttura schematica di un oggetto campione per esperimenti su rilevazione di Doppler vettoriale, costituito da un tubo di flusso principale con un innesto laterale di diametro più piccolo.

\section{\7. MEZZI DI CONTRASTO}

Lo sviluppo di nuove applicazioni degli ultrasuoni alla diagnosi medica è in continua evoluzione essendo elevato il potenziale di quantità di informazioni che sono raccolte dagli ultrasuoni nel processo di interazione con la materia ed in particolare con i tessuti biologici. A quanto sommariamente descritto nel presente lavoro si sono aggiunti i risultati preliminari che si sono ottenuti mediante l'uso dei mezzi di contrasto per ultrasuoni, costituiti da microbolle di pochi micron di diametro iniettati nel circolo sanguigno del corpo umano. Le microbolle danno luogo ad un segnale ultrasonico retrodiffuso che contiene spettri armonici e subarmonici generati da effetti di interazione di tipo non lineare (Fig. 22 e Fig. 23). L'uso dei mezzi di contrasto offre la possibilità di creare immagini selezionando solo il contenuto, ad esempio di seconda armonica; le immagini, di seconda armonica, quando si usino opportuni 
livelli di intensità ultrasonica tali da non innescare fenomeni non lineari nei tessuti biologici in esame, rappresentano solo il mezzo di contrasto che è presente nei vasi o nei tessuti, portato dai capillari sanguigni. Da tali immagini è possibile eseguire studi di perfusione del miocardio, localizzazione e delimitazione dei tumori nei quali il mezzo di contrasto è perfuso maggiormente nella porzione ricca di microvasi sanguigni di origine neoangiogenetica.

\section{DYNAMIC FLOW VISUALIZATION}

\section{Colour Flow Mapping 2-D Vector Doppler}

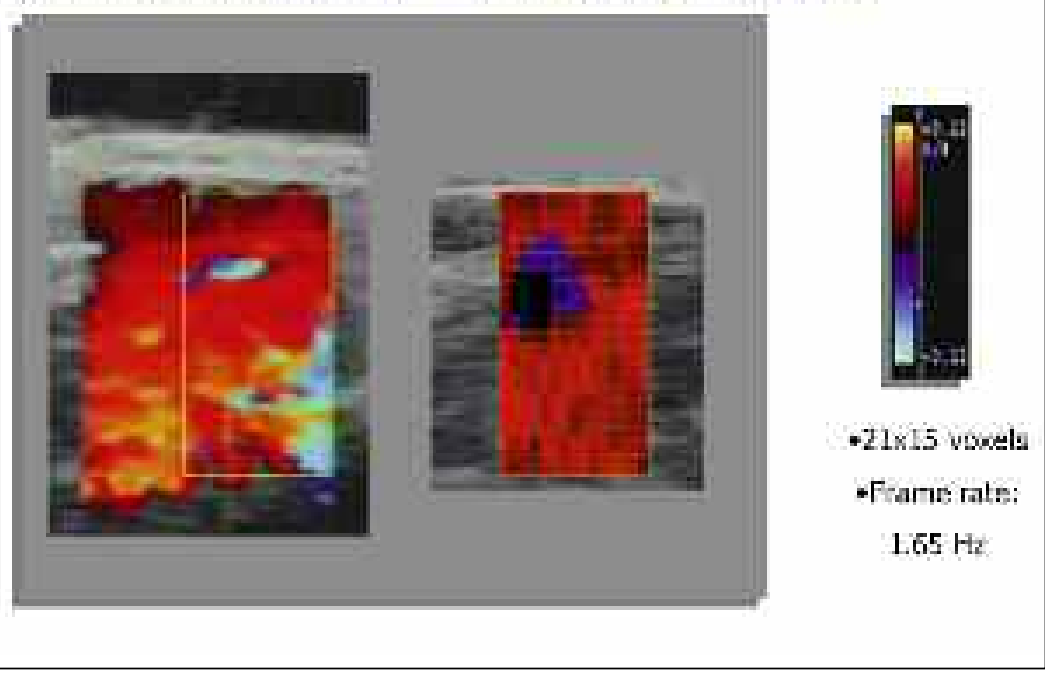

Fig. 22.

Rappresentazione di tipo B-mode con sovrapposto, a colori, l'andamento del Doppler vettoriale. La struttura sperimentale su cui sono state eseguite le prove è mostrata schematicamente in Figura 21. 


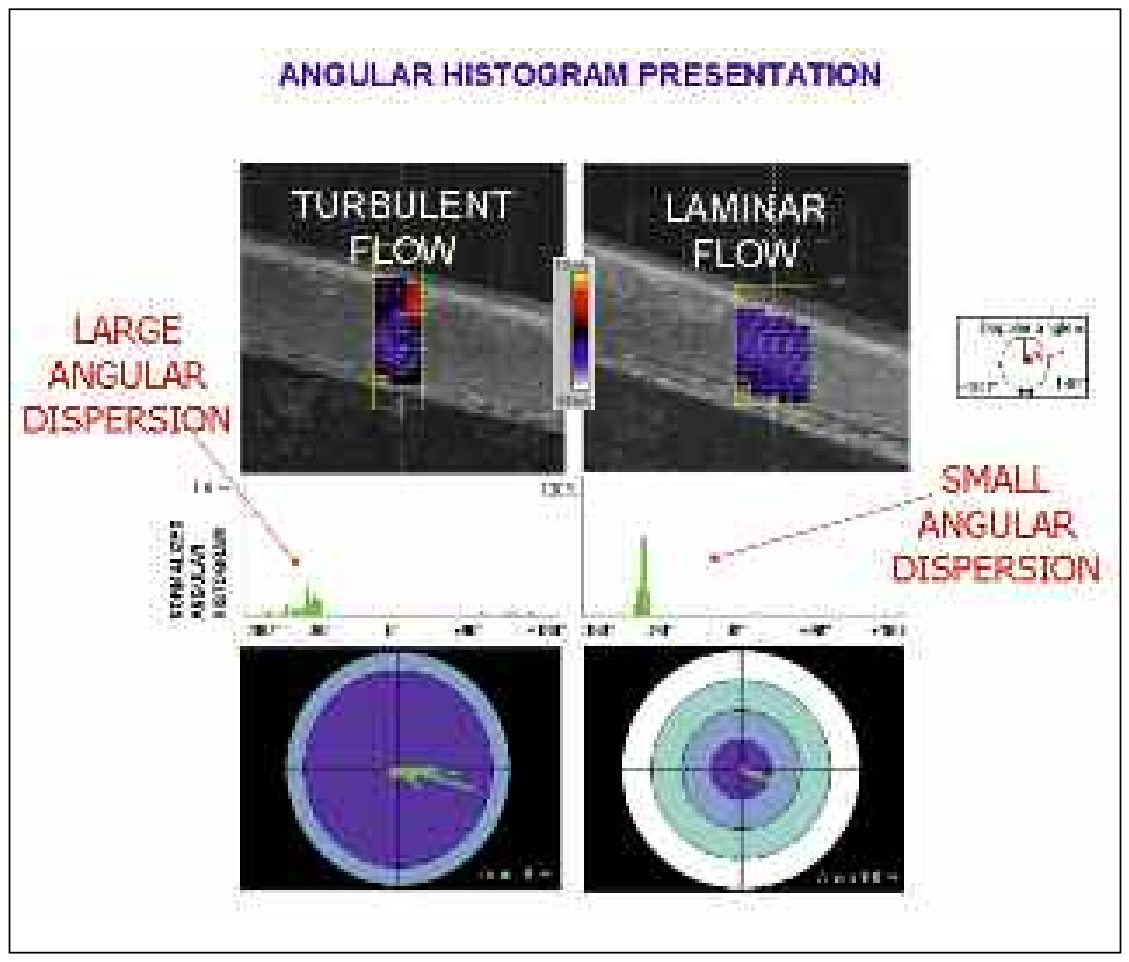

Fig. 23.

Andamento sperimentale dell'effetto Doppler su campione ottenuto in presenza di turbolenza e rappresentato a sinistra; a destra è rappresentato l'andamento per flusso in regime laminare.

\section{BIBLIOGRAFIA}

[1] L. Masotti, E. Biagi, A. Acquafresca, L. Breschi, M. Calzolai, R. Facchini, A. Giombetti, S. Granchi, A. Ricci, and M. Scabia. Ultrasonic images of tissue local power spectrum by means of wavelet packets for prostate cancer detection. Proc. Of the $26^{\text {th }}$ International Symposium Acoustical Imaging, In Press.

[2] E. Biagi, F. Margheri, and D. Menichelli. Efficient laser-ultrasound generation by using heavily absorbing films as targets. IEEE Trans. Ultrason. Ferroelect. Freq. Contr., S31, 2001. 
[3] D. Menichelli and E. Biagi. Optoacustic sources: a particular Green function-based model for thin film laser-ultrasound generation. J. Opt. A: Pure Appl. Opt., vol. 3, pp. S23-S31, 2001.

[4] M. Calzolai, L. Capineri, A. Fort, L. Masotti, S. Rocchi, and M. Scabia. A 3-D PW ultrasonic Doppler flowmeter: theory and experimental characterization. IEEE Trans. Ultrason. Ferroelect. Freq. Contr, vol. 46, no. 1, pp. 108-113, 1999.

[5] L. Capineri, M. Scabia, and L. Masotti. A Doppler system for dynamic vector velocity maps. Ultrason. Med. Biol. , vol. 28, pp. 237-248, 2002.

[6] R. Pini, E. Monnini, L. Masotti, KL Novins, DP Greenberg, B. Greppi, G. Cerofolini, M. Devereux. Echocardiographic three-dimensional visualization of the heart. Journal of the American College of Cardiology, 15:90A, IF:5.898, 1990. 